\title{
Isolated Hepatic Tuberculosis: Two Different Clinical Entities
}

\author{
Orhan Kursat Poyrazoglu ${ }^{\mathrm{a}, \mathrm{c}}$, Adile Ferda Dagli ${ }^{\mathrm{b}}$
}

\begin{abstract}
The diagnosis of tuberculosis is elusive because of its insidious nature and distinct clinical presentations. Isolated hepatic tuberculosis is a rare clinical entity among patients with tuberculosis. A 45 years old man was presented with complaints of upper abdominal pain, nausea and vomiting. Proteinuria induced by amyloidosis secondary to isolated hepatic tuberculosis was detected in routine urinalysis. Hepatic tuberculosis diagnosis was established by positive tuberculosis polymerase chain reaction from the liver tissue. A 27 years old man admitted to our hospital with a 2 months history of upper abdominal pain. The patient diagnosis was portal hypertension due to isolated hepatic tuberculosis. Both patients were followed up for 18 months under anti-tuberculosis treatment. Tuberculosis must be suspected in patients with liver mass in imaging studies and should be considered in the differential diagnoses that live in endemic areas for tuberculosis.
\end{abstract}

Keywords: Liver; Tuberculosis; Portal hypertension; Portal vein thrombosis; Amyloidosis

\section{Introduction}

Tuberculosis (TB) remains a serious public health problem in the worldwide because of increasing travel, migration and human immune deficiency virus infection. Although it is primarily considered a pulmonary disease, TB has the potential to infect almost every organ system. In addition, it is often overlooked because of its insidious nature, and the variability of its clinical presentation. Therefore, the diagnosis of TB needs high index suspicion to advocate potential morbidity and even

Manuscript accepted for publication April 15, 2015

a Department of Gastroenterology, School of Medicine, Firat University, Elazig, Turkey

${ }^{b}$ Department of Pathology, School of Medicine, Firat University, Elazig, Turkey

${ }^{\mathrm{c} C o r r e s p o n d i n g ~ A u t h o r: ~ O r h a n ~ K u r s a t ~ P o y r a z o g l u, ~ D e p a r t m e n t ~ o f ~ G a s t r o e n-~}$ terology, School of Medicine, Firat University, 23119 Elazig, Turkey.

Email: okpoyrazoglu@yahoo.com

doi: http://dx.doi.org/10.14740/jmc2140w mortality $[1,2]$. Hepatic TB is seen in less than $1 \%$ of all cases of TB [3]. It is frequently a part of miliary or pulmonary TB, and rarely presents as an isolated organ tuberculoma or tubercular abscess [4]. Here, we present two cases of hepatic TB, one of them with amyloidosis and the other one with portal vein thrombosis.

\section{Case Reports}

\section{Case 1}

A 45 years old man was admitted to our hospital with complaints of upper abdominal pain, nausea and vomiting. In addition, there was an 8 months history of high fever rising to 39 ${ }^{\circ} \mathrm{C}$, night sweat, loss of appetite and weight loss. On admission, his vital signs were normal. Bilateral peripheral edema was present $(+/+)$. The rest of the examination was unremarkable. Laboratory tests showed $\mathrm{Hb} 11 \mathrm{~g} / \mathrm{dL}$, Htc 33\%, WBC 13,000/ $\mathrm{mm}^{3}$, and PLT $486,000 / \mathrm{mm}^{3}$. Erythrocyte sedimentation rate was $107 \mathrm{~mm} / \mathrm{h}$. C-reactive protein was $60 \mathrm{mg} / \mathrm{dL}$. Liver function tests were normal except mildly elevated ALP $217 \mathrm{U} / \mathrm{L}$ (30 - 120) and GGT $139 \mathrm{U} / \mathrm{L}(0$ - 55). The serum albumin and total protein level were $2.2 \mathrm{~g} / \mathrm{dL}$ and $6 \mathrm{~g} / \mathrm{dL}$, respectively. Proteinuria $(+++)$ was detected in routine urinalysis. An electrocardiogram was normal.

Further tests revealed $5 \mathrm{~g}$ /day proteinuria in spot urinalysis. Tumor markers were within normal range and hepatitis markers were all negative. Multiple urine and blood cultures were negative. Amyloid accumulation was demonstrated histopathologically in rectal biopsy (Fig. 1A). Clinical, laboratory and histopathological findings strongly suggested a secondary amyloidosis. We performed PPD skin test that was $20 \times 20$ $\mathrm{mm}$. On radiological images, chest X-ray was normal. The abdominal ultrasound (US) showed $20 \times 25 \mathrm{~mm}$ hypoechoic lesion in the right lobe of the liver. A computed tomography (CT) scan revealed a single hypodense mass lesion (approximately $20 \mathrm{~mm}$ ) in the liver on segment 5 . There was a slightly peripheral enhancement following IV contrast administration (Fig. 1C). Tru-cut liver biopsy was performed under US guidance. TB polymerase chain reaction was positive from the liver biopsy specimen. Non-caseating granulomas were reported histopathologically and amyloid accumulation was also demonstrated (Fig. 1B). Anti-TB therapy including isoniazid, 


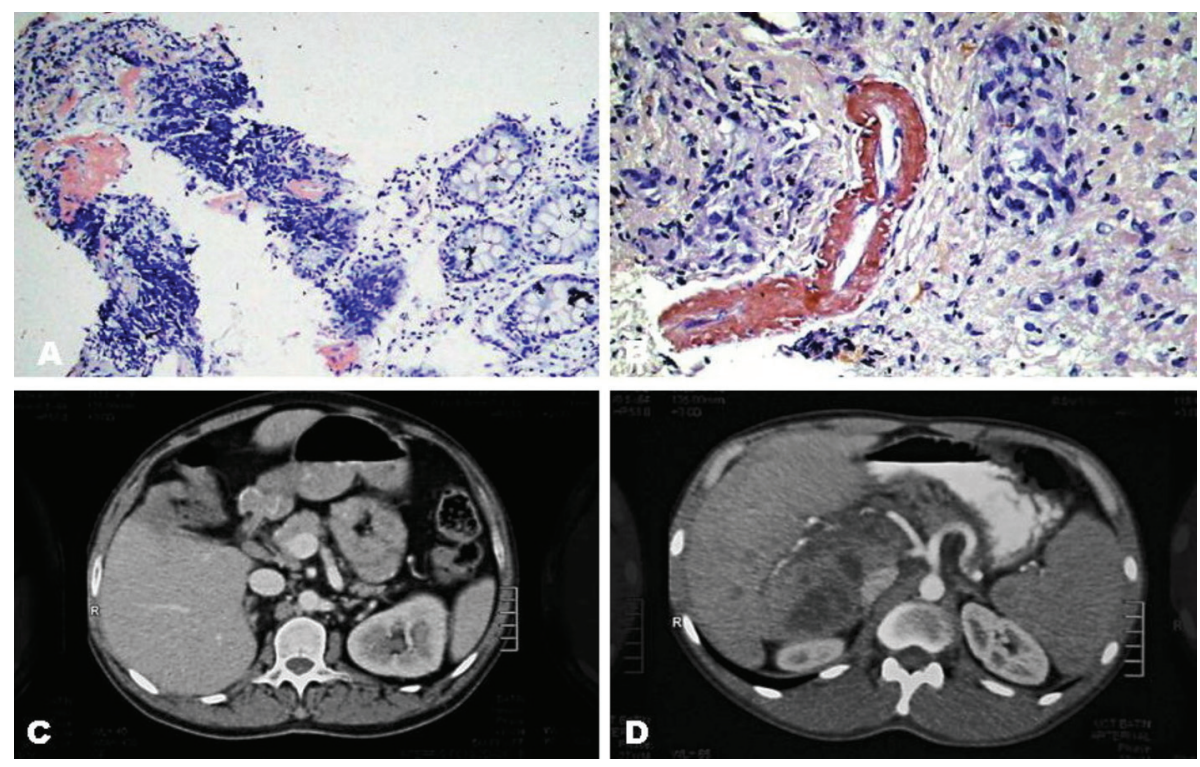

Figure 1. (A) Amyloid staining was positive in rectal biopsy (Kongo-Red, $\times 200$ ). (B) Amyloid staining was demonstrated at vascular wall in liver biopsy (Kongo-Red, $\times 200$ ). (C) Single hypodense mass lesion in the liver on segment 5 in case 1. (D) Hypodense mass lesions in the liver on segments 6 and 7 in case 2.

rifampicin, ethambutol (for first 2 months only) and pyrazinamid (for first 2 months only) was administered for 18 months. At the end of the follow-up, the patient was in good health, however, proteinuria remained $3 \mathrm{~g}$ /day in spot urinalysis.

\section{Case 2}

A 27 years old man presented to our hospital with a 2 months complaint of upper abdominal pain. There was no history of nausea, vomiting, loss of appetite, weight loss and fever. Physical examination revealed upper abdominal tenderness and splenomegaly. The rest of examination was within normal limits.

In laboratory evaluation, hematological profile was normal. Erythrocyte sedimentation rate was $23 \mathrm{~mm} / \mathrm{h}$. C-reactive protein was $117 \mathrm{mg} / \mathrm{dL}$. Liver function test showed elevated AST 44 U/L, ALT 155 U/L, ALP 160 U/L (30 - 120) and GGT $91 \mathrm{U} / \mathrm{L}(0-55)$. The serum albumin and total protein levels were in normal range. In addition, tumor markers were within normal range and hepatitis markers were all negative. PPD skin test was $20 \times 25 \mathrm{~mm}$.

On radiological images, chest X-ray was normal. The abdominal US showed $40 \times 30 \mathrm{~mm}$ and $30 \times 20 \mathrm{~mm}$ hypoechoic lesions in the right lobe of the liver. The longitudinal length of the spleen was $155 \mathrm{~mm}$. In addition, portal Doppler US showed thrombus as hyperechogenic lesion in portal vein. A CT scan revealed three hypodense mass lesions in the liver on segments 6 and 7 measuring $42 \times 33 \mathrm{~mm}, 20 \times 20 \mathrm{~mm}$ and $32 \times 23$ $\mathrm{mm}$ respectively. There was a slightly peripheral enhancement following IV contrast administration. The portal vein showed evidence of thrombus. In addition, enlarged lymph nodes were also noted among stomach, head of the pancreas and liver hilum (Fig. 1D). Tru-cut liver biopsy was performed under US guidance. Necroses were reported histopathologically. Anti-TB therapy including isoniazid, rifampicin, ethambutol (for first 2 months only) and pyrazinamide (for first 2 months only) was administered for 18 months. In addition, anticoagulant therapy was also started. At the end of the follow-up, the patient was in good health, albeit persistence of portal vein thrombosis.

\section{Discussion}

Abdominal TB usually involves the peritoneum, gastrointestinal tract, abdominal lymph nodes and solid visceral organs. Among extrapulmonary TB, hepatic TB is considered to be a rare clinical entity. There are multiple manifestations of liver TB [3-5]. Hepatic TB has been classified as military TB, pulmonary TB with liver involvement, primary liver TB, isolated tuberculoma and tuberculous cholangitis $[2,3]$. The miliary form is the most common and spreads mainly through hematogenous dissemination.

Hepatic TB can be presented by various clinical findings. Therefore, the clinician must be aware of its presentation to reach the correct diagnosis. The most frequent clinical and laboratory findings in localized hepatic TB are fever, weight loss, night sweat, fatigue, abdominal pain, hepatomegaly, jaundice, anemia, raised ESR, abnormal hepatic function and positive tuberculin test [3]. In addition, some patients can present with nephrotic syndrome due to TB as in case 1. During long follow-up of patients with amyloid nephropathy clinical remission may be observed. However, remission is rare, independent of etiology and type of amyloidosis [6] as observed in our case 1.

Radiological diagnosis of the hepatic TB frequently remains a diagnostic challenge. Macronoduler hepatic tuberculomas, which measure more than a few centimeters, are rare. 
The lesions can be single or multiple [5, 7-9]. The US appearance of the lesions is hypoechoic or rarely hyperechoic. The CT findings of the macronodular tuberculomas are considered variable and non-specific. Appearance of the lesion varies from hypodense mass, with or without rim enhancement after contrast, to a heterogeneous density of the necrotic center of bull's eye calcifications. Magnetic resonance imaging adds no more information to US and CT findings. Chest X-ray may reveal abnormal lung lesions in $10-86 \%$ of the patients $[3,8]$. Therefore, the absence of pulmonary disease cannot be excluded in the diagnosis of hepatic TB. Our patients presented with no evidence of active pulmonary diseases. In addition, the US and CT appearance of the patients confirmed the findings mentioned above. On the other hand, a thrombus was also seen in the right branch of portal vein that is suggesting malignant lesion in case 2 as recorded before [4, 9]. Abdominal TB is one of the causative factors of portal vein thrombosis that results with esophageal and gastric varices [4, 8]. Our patient was treated with anticoagulant therapy, however, recanalization was not achieved completely.

Although the radiological findings of hepatic tuberculosis help to establish the diagnosis, its low specificity requires microbiological, pathological and clinical confirmation. Thereby, we can distinguish the primary or metastatic carcinoma of the liver, pyogenic or amebic liver abscess in light of these diagnostic tools. Alcantara-Payaway et al [10] developed a PCR assay for the identification Mycobacterium tuberculosis in liver biopsy specimens. Histopathologically, caseating granuloma, non-caseating granuloma or caseous necrosis in the liver biopsy may reveal the diagnosis of TB. Other non-specific histological findings include Kupffer cell hyperplasia, focal hepatocyte necrosis with round cell infiltration and portal inflammation $[2,3,6]$. In the differential diagnosis of TB, primary biliary cirrhosis, Crohn's disease, chronic active hepatitis, drug hypersensitivity and extrahepatic biliary obstruction should be rule out. Therefore, to confirm the diagnosis for TB, clinical picture of the patient, additional laboratory findings such as a positive AFB, culture, PCR and clinical response to anti-TB therapy should be taken into consideration. PCR provided the diagnostic evidence in case 1. However, the diagnosis of TB was confirmed with response to anti-TB therapy in case 2 .

Treatment of hepatic TB includes the standard four-drug regimen with isoniazid, rifampicin, ethambutol and pyrazinamide as for any other form of extrapulmonary TB. In addition, the duration of therapy is recommended generally for 1 year. Clinical improvement is frequently seen within $2-3$ months. Surgery is an option for multiple large lesions and lesions refractory to medical treatment $[2,3]$. In our two cases, the duration of therapy was 18 months, however, symptom relief was achieved within 2 months. In addition, surgery is not required.

In conclusion, hepatic TB must be suspected in patients with upper abdominal pain, fever, weight loss, and liver mass in imaging studies and should also be considered in the differential diagnosis that live in endemic areas for TB. On the other hand, physicians should keep in mind that patients with hepatic TB can present with amyloidosis and portal vein thrombosis. Although microbiological and pathological examination adds on further information in patient with liver mass to establish the diagnosis of hepatic TB, liver biopsy should be performed to rule out malignancy.

\section{References}

1. Braun MM, Byers RH, Heyward WL, Ciesielski CA, Bloch AB, Berkelman RL, Snider DE. Acquired immunodeficiency syndrome and extrapulmonary tuberculosis in the United States. Arch Intern Med. 1990;150(9):19131916.

2. Poyrazoglu OK, Timurkaan M, Yalniz M, Ataseven H, Dogukan M, Bahcecioglu IH. Clinical review of 23 patients with tuberculous peritonitis: presenting features and diagnosis. J Dig Dis. 2008;9(3):170-174.

3. Mert A, Ozaras R, Tabak F, Ozturk R, Bilir M. Localized hepatic tuberculosis. Eur J Intern Med. 2003;14(8):511512.

4. Venkatesh SK, Tan LK, Siew EP, Putti TC. Macronodular hepatic tuberculosis associated with portal vein thrombosis and portal hypertension. Australas Radiol. 2005;49(4):322-324.

5. Farooq Sheikh AS, Qureshi IH, Saba K, Bukhari MH. Primary isolated hepatic tuberculosis. J Coll Physicians Surg Pak. 2013;23(5):359-361.

6. Kaaroud H, Boubaker K, Beji S, Abderrahim E, Moussa FB, Turki S, Goucha R, et al. Renal amyloidosis followed more than 5 years: report of 12 cases. Transplant Proc. 2004;36(6):1796-1798.

7. Karunanithi S, Sharma P, Jain TK, Vijay MK, Kumar R. Multiple hepatic lesions in a case of isolated hepatic tuberculosis simulating metastases on 18F-FDG PET/CT imaging. Nucl Med Rev Cent East Eur. 2014;17(2):108109.

8. Akhan O, Pringot J. Imaging of abdominal tuberculosis. Eur Radiol. 2002;12(2):312-323.

9. Hung YM, Huang NC, Wang JS, Wann SR. Isolated hepatic tuberculosis mimicking liver tumors in a dialysis patient. Hemodial Int. 2015;19(2):344-346.

10. Alcantara-Payawal DE, Matsumura M, Shiratori Y, Okudaira T, Gonzalez R, Lopez RA, Sollano JD, et al. Direct detection of Mycobacterium tuberculosis using polymerase chain reaction assay among patients with hepatic granuloma. J Hepatol. 1997;27(4):620-627. 